\title{
Nonlinear Analysis and Optimization
}

\author{
Alexey F. Izmailov ${ }^{1}$ • Fernando Lobo Pereira ${ }^{2}$. Boris S. Mordukhovich ${ }^{3}$ \\ Published online: 22 November 2018 \\ (c) Springer Science+Business Media, LLC, part of Springer Nature 2018
}

\begin{abstract}
This is the Preface to the Special Issue of JOTA dedicated to the 60th birthday of Professor Aram V. Arutyunov
\end{abstract}

Keywords Nonlinear and variational analysis · Optimization · Optimal control

Mathematics Subject Classification 47H10 - 49J15 - 49J20 • 49J53 · 90C20

Aram Vladimirovich Arutyunov is a major player in nonlinear analysis, optimization theory, and optimal control. This Special Issue of JOTA is dedicated to his great achievements in those and related areas over the years. It follows the International Workshop on Nonlinear Analysis and Optimization Theory held in Porto, Portugal in April of 2017, which was dedicated to Aram's 60th birthday.

Aram Arutyunov graduated from Moscow State University, where he is now a professor of Applied Mathematics jointly with his professorship at the RUDN University of Moscow. Aram's first interests were in the area of optimal control, and these topics continue to be at the heart of his research. His achievements in control theory are difficult to overstate. Among them, we list fundamental results on the Pontryagin Maximum Principle for control problems with state and mixed constraints, deep investigations of abnormal extremals, new controllability conditions to avoid the degeneracy phenomenon, second-order optimality conditions for singular and nonsingular extremals, necessary optimality conditions for Lipschitzian differ-

\footnotetext{
$\bowtie$ Boris S. Mordukhovich boris@math.wayne.edu

Alexey F. Izmailov izmaf@cs.msu.ru

Fernando Lobo Pereira flp@fe.up.pt

1 Faculty of Computational Mathematics and Cybernetics, Moscow State University, Leninskiye Gori, GSP-2, Moscow, Russia 119992

2 FEUP/DEEC, University of Porto, Rua Dr. Roberto Frias, 4200-465 Porto, Portugal

3 Department of Mathematics, Wayne State University, Detroit, MI 48202, USA
} 
ential inclusions and nonsmooth control systems governed by ordinary differential equations, theory of degenerate quadratic form in the classical calculus of variations, novel approaches and results on impulsive optimal control, etc. These results rely on the new mathematical tools developed by Aram.

Among various achievements of Prof. Arutyunov and his collaborators in general optimization theory for problems with no dynamic structures, we particularly mention very strong results on sensitivity analysis of feasible and optimal solutions. Series of Arutyunov's papers develop a number of striking and rather surprising higherorder "root" estimates for non-Lipschitzian stability in constrained optimization in the absence of standard constraint qualifications. Perhaps the most striking Aram's contribution to optimization consists of developing the theory of first-order and second-order necessary and sufficient optimality conditions for abnormal optimization problems with various constraints in Banach spaces.

Concerning the problems of broadly understood modern nonlinear analysis, which may and may not involve optimization aspects, there are breakthrough achievements that have been reached by Aram Arutyunov over the years. Here, we cannot miss mentioning fundamental results by Aram on fixed points and coincidence points of mappings between general metric spaces, partially ordered spaces as well as their generalizations and specifications, his beautiful and powerful theory of quadratic mappings, inverse and implicit function theorems, and impressive novel developments on directional metric regularity and covering concepts that play a crucial role in setvalued and variational analysis. Some of the results mentioned above were obtained by Aram in collaboration with his former PhD students Dmitry Karamzin and Sergey Zhukovskiy.

This special issue includes 16 papers devoted to various aspects of nonlinear analysis, optimization, optimal control, and applications that are mainly connected with Aram Arutyunov's research. We briefly describe below some characteristic aspects of each paper included in the issue.

The paper by Adrian Petrusel, Gabriela Petrusel and Jen-Chih Yao contains new fixed point theorems for single-valued and set-valued mappings on quasimetric spaces. The developed approach is based on the concept of metric regularity and advanced tools of modern variational analysis.

The paper by Simeon Reich and Alexander Zaslavski also deals with fixed points while for nonlinear operators of a contractive type defined on complete metric spaces. The obtained results establish not only the existence of fixed points and the convergence of successive approximations, but also provide information on behavior of inexact orbits.

Tatiana Fomenko and Dmitrii Podoprikhin study in their paper fixed point properties of mappings on ordered sets. They extend several results on preservation of fixed points under a homotopy to common fixed points of families of mappings in ordered sets.

The paper by Aram Arutyunov, Evgeny Zhukovskiy and Sergey Zhukovskiy concerns nonlinear mappings on partially ordered spaces under a certain Caristi-like condition. The authors obtain impressive results on the existence of minimal elements for such mappings and apply them to developing new theorems on the existence of coincidence points of mappings between partially order spaces. 
Samir Adly develops in his paper a coderivative approach to Lipschitzian stability of solutions maps to parametric composite variational inequalities. He employs advanced tools of first-order and second-order variational analysis and generalized differentiation with applications to models of nonsmooth mechanics.

The two interrelated papers by Duong Thi Kim Huyen, Jen-Chih Yao and Nguyen Dong Yen are devoted to sensitivity analysis of stationary solution maps associated with perturbed smooth problems of constrained optimization. The first paper deals with Lipschitzian stability, while the second part concerns Robinson stability. The authors obtain necessary and sufficient conditions for these properties by using the coderivative criterion and second-order subdifferential calculus of variational analysis.

The paper by Andreas Fischer, Alexey Izmailov and Mikhail Solodov addresses several Newton-type methods to solve constrained equations and complementarity problems. The main attention is paid to the situations where a given solution is singular and even not isolated. Developing a novel technique, the authors establish the linear convergence of iteration sequences to the solution in question.

Nicolas Hadjisavvas, Felipe Lara and Juan Enrique Martinez-Legaz introduce in their paper a new notion of asymptotic functions and study this notion in detail with particular relationships to quasiconvexity. Then, they apply the established properties of asymptotic functions to quasiconvex optimization.

The paper by Alfredo Iusem and Felipe Lara elaborates on sufficient conditions for a solution set of noncoercive pseudomonotone equilibrium and vector equilibrium problems to be nonempty and compact. By using asymptotic analysis, the authors also characterize efficient and weakly efficient solutions to nonconvex vector equilibrium problems via a scalarization technique.

In their paper, Francesca Chittaro and Laura Poggiolini show that Pontryagin extremals of optimal control problems with dynamics affine in control variables and a cost functional involving the absolute value of the control are given by (possible) concatenations of bang arcs, singular arcs, and inactivated arcs. Under certain regularity and coercivity conditions, sufficient conditions for such extremals are derived by using a Hamiltonian approach.

The paper by Dmitry Karamzin and Fernando Lobo Pereira refine necessary optimality conditions in the Pontryagin Maximum Principle form for optimal control problems with state constraints in order to ensure the nondegeneracy of the conditions under weaker controllability assumptions and, moreover, to establish the continuity of the measured Lagrange multiplier.

Nguyen Hoang and Boris Mordukhovich address in their paper a new class of optimal control problems for sweeping/Moreau processes governed by discontinuous unbounded differential inclusions that are specified via the normal cone to controlled moving sets. Nondegenerate necessary optimality conditions in the forms of extended Euler-Lagrange and, for the first time for such systems, of a novel form of the Maximum Principle are derived by developing the method of discrete approximations and generalized differential tools for first-order and second-order variational analysis.

The paper by Andrei Faminskii deals with PDE control problems governed by the Korteweg-de Vries equation on the infinite horizon with a certain integral constraint. The author obtained sufficient conditions for the existence and uniqueness of 
an unknown control function on the right-hand side of the equation that ensures the validity of the integral constraint for the corresponding state trajectory.

The paper by Rovshan Bandaliyev, Vagif Guliyev, Ilgar Mamedov, and Yasin Rustamov also belongs to the area of PDE control while being governed by the third-order Bianchi equation with nonclassical Goursat-type boundary conditions. By developing an appropriate version of the increment method, the authors obtained necessary and sufficient optimality condition in a Maximum Principle form.

The last paper included in this SI is by Urszula Ledzewicz, Helmut Maurer and Heinz Schaettler on applications of optimal control. The authors develop an optimal control model of combined radio- and antiangiogenic therapy, which is governed by ordinary differential inclusions with minimizing the tumor volume under isoperimetric constraints. Besides further improving their previous results based on the characterization of the extremals, they provide various examples of practical meaning to confirm their analytical conclusions.

Finally, the Guest Editors are happy to extend their congratulations to Aram Arutyunov on his great achievements, to thank all the authors for their excellent contributions.

Acknowledgements The first author was supported in part by the Russian Foundation for Basic Research Grant 17-01-00125 and by Volkswagen Foundation. The second author was partially supported by FCT R\&D Unit SYSTEC-POCI-01-0145-FEDER-006933/ERDFICOMPETE2020|FCT/MECIPT2020, and Project STRIDE-NORTE-01-0145-FEDER-000033 funded by ERDFINORTE 2020. Work of the third author was partly supported by the USA National Science Foundation under grants DMS-1512846 and DMS-1808978, and by the USA Air Force Office of Scientific Research under Grant \#15RT0462. 